\title{
Spectroscopie laser femtoseconde dans l'eau, milieu biologique
}

\author{
J.-C. Mialocq, T. Gustavsson et S. Pommeret
}

CEA Saclay, DSMIDRECAM/SCM, URA 331 du CNRS, 91191 Gif-sur-Yvette cedex, France

\begin{abstract}
Résumé : Les applications des impulsions laser ultra-brèves et accordables en longueur d'onde de l'IR à l'UV constituent des domaines exploratoires actifs et fructueux pour l'étude des processus primaires uitrarapides en photochimie et en photobiologie. La génération des impulsions laser femtoseconde et deux techniques de spectroscopie résolue en temps en fluorescence et en absorption sont brièvement présentées. La génération en optique non-linéaire de la somme de fréquences dans des cristaux permet de sonder à l'échelle de temps femtoseconde la fluorescence des états excités, délivrant des clichés instantanés de la couleur de la fluorescence, révélateurs des propriétés des molécules, de leur micro-environnement, et des vitesses des processus intra- et intermoléculaires. La spectroscopie d'absorption pompe-sonde qui renseigne sur le peuplement et le dépeuplement des états fondamental et excités des molécules est particulièrement utile quand les processus sont non-radiatifs. Quelques applications concernant les processus de transfert de charge intramoléculaire, de transfert d'électron au solvant, de relaxation intra- et inter-moléculaire illustreront les progrès récents des connaissances sur les processus ultrarapides dans l'ean, milieu biologique.
\end{abstract}

\section{INTRODUCTION}

Les progrès dans la génération et les diagnostics des impulsions laser pico- et femto-seconde accordables en longueur d'onde de l'IR à l'UV et le développement des méthodes de détection et d'acquisition des données expérimentales [1] permettent des études spectroscopiques et cinétiques en fluorescence et en absorption, toujours plus fines et indispensables pour comprendre les processus primaires ultrarapides en photochimie et en photobiologie. Dans de nombreux photo-systèmes artificiels ou naturels, on peut suivre aujourd'hui la dynamique des processus de l'échelle femtoseconde à l'échelle milliseconde. Dans les rhodopsines par exemple [2,3], photo-récepteurs rencontrés dans la nature, le rétinal lié le plus souvent à une protéine membranaire subit une photoisomérisation responsable (du fait des rotations et des contraintes) du transfert de l'énergie libre en excès à la protéine, cause de changements de $\mathrm{pK}$ des résidus amino-acides et de transferts de proton. Comprendre le rôle de l'eau dans la réactivité demeure difficile même si on connâit quelques règles semi-empiriques où la polarité, la polarisabilité et le caractère donneuraccepteur de líaison hydrogène du solvant rendent compte des interactions soluté-solvant, des déplacements des spectres d'absorption et de fluorescence des molécules et des vitesses de réaction. Les variations d'énergie libre et d'énergie d'activation des réactions dépendent en effet comme les déplacements spectraux, des énergies de solvatation des réactifs, des produits et de l'état de transition, d'où l'intérêt de l'analyse des spectres des espèces chimiques à l'échelle femtoseconde. Une approche fructueuse est l'analyse temporelle des déplacements des spectres de fluorescence, d'émission stimulée et d'absorption des états électroniques excités de molécules porteuses d'un groupe donneur et d'un groupe accepteur d'électron, telles que les aminocoumarines et le système anion ferrocyanure-eau étudiés dans ce travail. En effet, l'excitation lumineuse des aminocoumarines induit un transfert de charge intra-moléculaire qui perturbe l'équilibre de solvatation. Le nuage électronique du soluté et la polarisation induite du solvant ont une réponse ultra-rapide $\left(<10^{-15} \mathrm{~s}\right)$ tandis que les noyaux et la "cage de solvant" immobiles à cette échelle de temps (Principe de Franck-Condon) se relaxent plus lentement $\left(10^{-14}-10^{-11} \mathrm{~s}\right.$ ). En revanche, l'excitation lumineuse dans l'ultraviolet du ferrocyanure provoque une réaction efficace et ultrarapide $\left(<50 \times 10^{-15} \mathrm{~s}\right) \mathrm{de}$ photo-détachement d'un électron. L'électron s'hydrate ultérieurement à l'échelle subpicoseconde. 


\section{SPECTROSCOPIE DE FLUORESCENCE FEMTOSECONDE. PROCESSUS INTRA- ET INTER-MOLECULATRES}

Les mesures de fluorescence à la fois dans le domaine temporel et dans le domaine spectral sont certainement les plus faciles à interpréter car la fluorescence est le plus souvent émise par des molécules portées dans un seul état électronique excité. Ces mesures apportent des informations sur les vitesses des processus intra- et inter-moléculaires, sur les interactions soluté-solvant et sur les relations structureréactivité. Au delà de la dizaine de picosecondes, la technique largement répandue du comptage de photons uniques permet d'analyser la montée et le déclin de l'intensité de fluorescence des molécules sur plusieurs décades en intensité. A l'échelle de temps femtoseconde et jusqu'à 200 picosecondes environ après l'excitation lumineuse, la technique optique non-linéaire de la génération de la somme de fréquences dans des cristaux permet de sonder la fluorescence des états excités avec une résolution temporelle donnée par la durée de 1'impulsion laser (Figure 1) [4]. Elle délivre des clichés instantanés de la couleur de la fluorescence, révélateurs des propriétés intrinsèques des molécules, de leur micro-environnement et de la dissipation de l'énergie en excès du système chimique ou biologique.

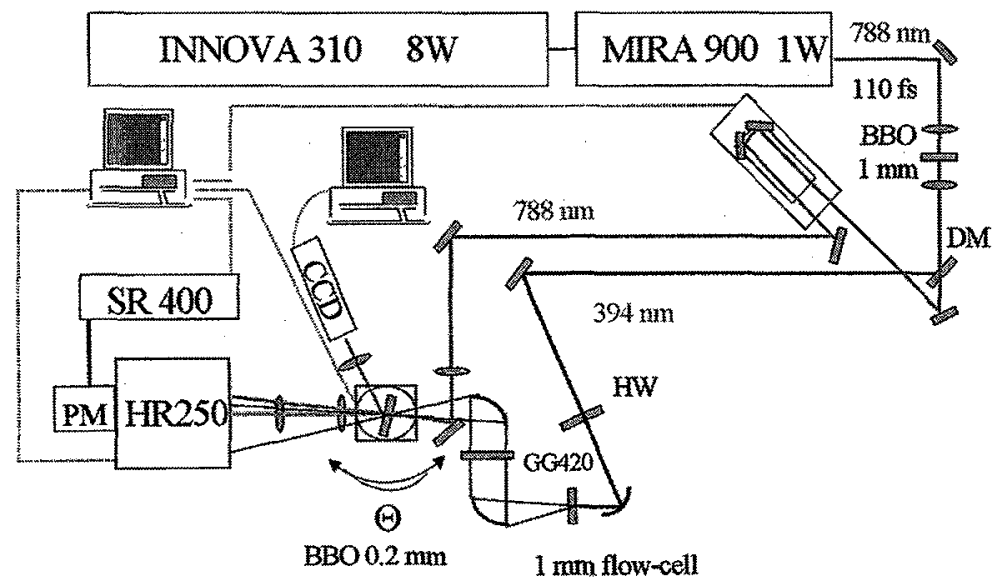

Figure 1 : Montage du spectrofluorimètre femtoseconde par conversion de fréquence

Les coumarines solubles dans l'eau ont des applications très importantes en biologie (marqueurs fluorescents) et dans les lasers à colorant. Comme colorants laser, les 7-aminocoumarines sont généralement mises en solution dans des solvants organiques, le méthanol, l'éthanol, voire le dioxane qui sont toxiques, inflammables ou à faible limite d'explosivité et qui présentent d'autres inconvénients majeurs : leurs indices de réfraction dépendent fortement de la température, ils ont de faibles coefficients de transfert de chaleur. Les solutions aqueuses seraient donc préférables.

Il s'avère que l'efficacité des processus non-radiatifs de relaxation du premier état singulet excité des 7-aminocoumarines commerciales $(\mathrm{C} 120, \mathrm{C} 311, \mathrm{C} 1$ et $\mathrm{C102})$ dépend fortement de la nature des substituants $R(H, M e, E t)$ du groupement amino en position 7 , de celle du substituant $R^{\prime}$ en position 4 $\left(\mathrm{CH}_{3}, \mathrm{CF}_{3}\right)$ et du solvant. Substituées par des groupes polyéthers en position 4 (ATC, DMATC, DATC et COHS), elles sont solubles dans l'eau. Leurs propriétés spectrales, leurs rendements quantiques et durées de vie de fluorescence ont été étudiés dans divers solvants pour analyser les interactions soluté-solvant dans les états électroniques singulets fondamental et excité [4]. Ces coumarines sont des molécules à transfert de charge dont l'état électronique singulet excité est plus polaire que l'état fondamental. Il est donc plus sensible à la polarité du solvant. Elles ont des rendements quantiques de fluorescence élevés et de longues durées de vie (quelques ns) dans la plupart des solvants, mais pour certaines d'entre elles, comme la 7-diéthylamino-4-méthylcoumarine (C1) et son analogue, la DATC, ces valeurs chutent considérablement à cause d'un processus non-radiatif de relaxation totalement incompris à ce jour.

Par l'étude de la relaxation du premier état singulet excité de ces coumarines on a cherché à faire la part entre les processus intramoléculaires et les processus intermoléculaires (solvatation). Les coumarines hydrosolubles nous permettent de sonder la dynamique de l'eau. On a aussi étudié d'autres solvants polaires, protiques et aprotiques pour différencier les rôles respectifs de la polarité/polarisabilité et des 
liaisons hydrogène. La stabilisation du premier état excité semble être parfaitement contrôlée par la réponse du solvant (quelques picosecondes), même pour la coumarine $\mathrm{C} 1$ qui manifeste une voie de désactivation efficace [5]. Il n'y a qu'un seul état fluorescent dans tous les solvants sur l'échelle de quelques centaines de picosecondes, ce qui montre que l'on peut écarter l'hypothèse de la formation rapide d'un état "TICT". En effet, le caractère très homogène de la relaxation aux temps courts indique que la dynamique de solvatation, processus dominant, n'est pas couplée avec le processus responsable du "quenching" de fluorescence.

A titre d'exemple, les déplacements de Stokes $v(t)-v(\infty)$ au cours du temps des spectres de fluorescence de $\mathrm{C} 1$ dans l'eau et l'eau lourde sont présentés dans la figure 2. Les valeurs sont calculées à partir des fréquences moyennes des spectres de fluorescence après déconvolution pour la fonction d'appareil (185 fs FWHM). Dans ces deux solvants, la cinétique du déplacement est un déclin triexponentiel mais les différentes composantes diffèrent. Une première composante dont la constante de temps $\tau_{1}$ a été fixée à 50 fs dans les deux cas, compte pour 50-60\% du déplacement total. Regroupant tous les contributions trop rapides pour être analysées avec notre résolution temporelle de $50 \mathrm{fs}$, cette composante ultra-rapide est dominée par la relaxation intra-moléculaire (vibronique) mais une contribution de la solvation inertielle ne peut pas être exclue ni une contribution des raies Raman. Elle est suivie d'une deuxième composante deux fois plus lente dans l'eau lourde $\left(\tau_{2}=1,5 \mathrm{ps}\right)$ que dans l'eau légère $\left(\tau_{2}=750\right.$ fs). Une troisième composante de constante de temps $\tau_{3}$ voisine de 10 picosecondes représente 3 à $5 \%$ du déplacement total. Néanmoins, la faible précision des paramètres de cette dernière composante a peu d'effet sur la valeur de $\tau_{2}$. Le rapport 2 entre les valeurs de $\tau_{2}$ mesurées dans l'eau légère et dans l'eau lourde attribué à l'effet isotopique H/D est l'objet d'un examen plus approfondi.

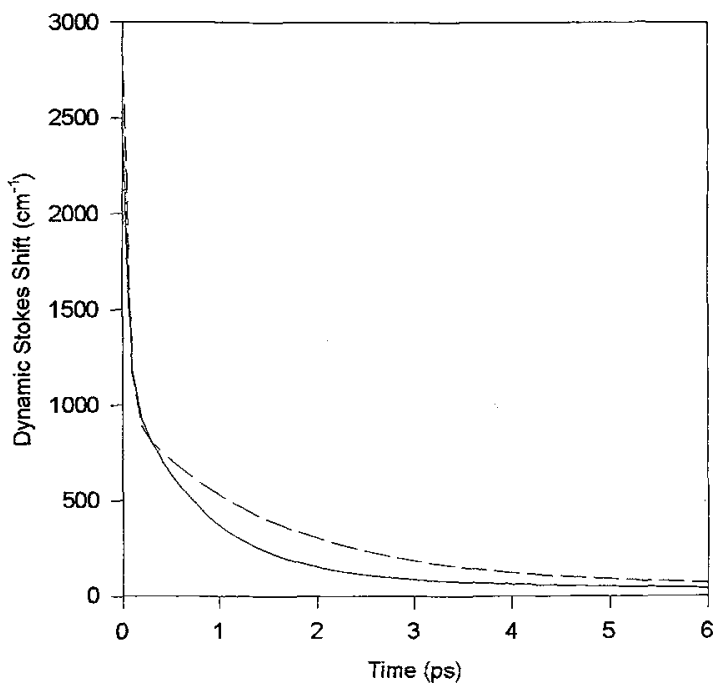

Figure 2: Déplacements de Stokes des spectres de fluorescence de la coumarine $\mathrm{Cl}$ dans l'eau légère et dans l'eau lourde.

\section{SPECTROSCOPIE D'ABSORPTION FEMTOSECONDE. PHOTODETACHEMENT DE L'ELECTRON}

La spectroscopie d'absorption femtoseconde est complémentaire de la fluorescence car elle permet de décrire en plus les transitions non-radiatives des états électroniques excités. Cependant en spectroscopie d'absorption pompe-sonde, les données sont plus délicates à analyser parce que de nombreuses bandes d'absorption ou de gain des molécules portées dans des états électroniques excités ou retournant à l'état fondamental contribuent au signal détecté. Les fréquences de répétition des impulsions laser qui atteignent maintenant couramment le kilohertz assurent l'enregistrement des spectres d'absorption résolus en temps avec un excellent rapport signal/bruit. 
Notre chaîne laser kilohertz à saphir dopé au titane et cadencée à $1 \mathrm{kHz}$, décrite précédemment [6,7], fournit à $800 \mathrm{~nm}$ des impulsions d'une durée inférieure à $50 \mathrm{fs}$ et d'une énergie de $750 \mu \mathrm{J}$. La fonction de réponse de notre spectrophotomètre d'absorption est bien approchée par une gaussienne dont la largeur à mi-hauteur est de $60 \mathrm{fs}$. Dans une étude récente sur le photodétachement de l'électron à partir de l'ion ferrocyanure à $266 \mathrm{~nm}$ (3ème harmonique), nous avons pu mettre en évidence l'apparition de l'électron hydraté avec une constante de temps de 510 fs et comparer les cinétiques observées à celles trouvées dans la littérature [6]. Ici nous présentons des résultats nouveaux obtenus dans le proche infrarouge (Figure 3).

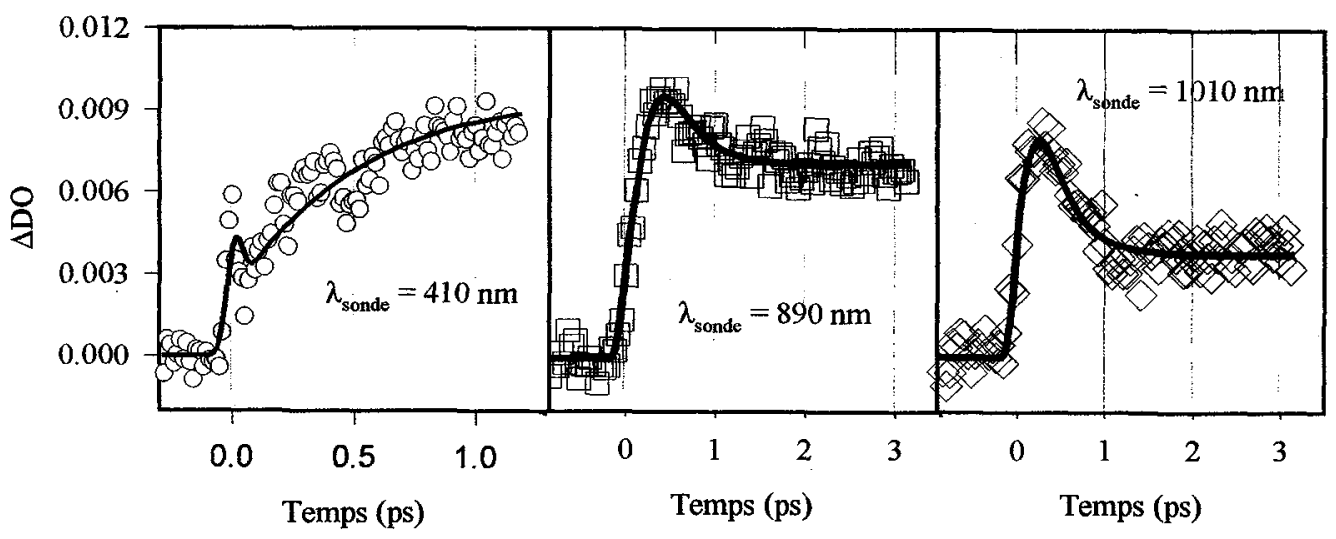

Figure 3 : Absorption transitoire consécutive à la photoexcitation à $266 \mathrm{~nm}$ par une impulsion laser femtoseconde d"une solution aqueuse $0,01 \mathrm{M}$ de ferrocyanure de potassium en cellule de silice de $0,13 \mathrm{~mm}$ de parcours optique.

Les données obtenues dans le proche infrarouge montrent la présence d'une espèce à courte durée de vie, formée avec une constante de temps de $190 \mathrm{fs}$. Elle disparait en donnant l'électron hydraté avec une constante de temps de $300 \mathrm{fs}$. Dans cette expérience, l'excitation lumineuse du ferrocyanure est monophotonique dans une bande de transfert de charge au solvant.

Avec le développement de chaines laser femtoseconde avec amplification paramétrique optique qui permettent l'accordabilité de l'excitation laser, on peut s'attendre dans les prochaines années, à des progrès importants dans la compréhension des mécanismes conduisant à la formation de l'électron hydraté, espèce réductrice d'une grande importance en chimie et en biologie.

\section{Références}

1. W. Sibbett, CLEO/EUROPE-EQEC'98, Glasgow, Scotland, 14-18 September 1998

2. Q. Wang, G.G. Kochendoerfer, R.W. Schoenlein, P.J.E. Verdegem, J. Lugtenburg, R.A. Mathies et C.V. Shank, J. Phys. Chem. 100, 17388 (1996)

3. T. Kobayashi, M. Kim, M. Taiji, T. Iwasa, M. Nakagawa et M. Tsuda, J. Phys. Chem. B 102, 272 (1998)

4. T. Gustavsson, L. Cassara, V. Gulbinas, G. Gurzadyan, J.-C. Mialocq, S. Pommeret, M. Sorgius et P. v.d. Meulen, J. Phys. Chem. A 102, 4229 (1998)

5. L. Cassara, T. Gustavsson, S. Pommeret, J.-C. Mialocq, J. Chalom et A. Reveilleau, Fast Elementary Processes in Chemical and Biological Systems, AIP Conference Proceedings 364, 1996, pp. 486-495.

6. S. Pommeret, R. Naskrecki, P. v.d. Meulen, M. Ménard, G. Vigneron et T. Gustavsson, Chem. Phys. Lett. 288, 833 (1998) et ses références.

7. R. Naskrecki, M. Ménard, P. v.d. Meulen, G. Vigneron et S. Pommeret, Opt. Comm. 135, 32 (1998) 